\section{P1-524 COMPARATIVE STUDY OF THE INFLUENZA PANDEMIC OF 1918-1919 IN TWO ISLAND NATIONS: ICELAND AND NEW ZEALAND}

\author{
doi:10.1136/jech.2011.142976h.12
}

${ }^{1} \mathrm{~J}$ A Summers, ${ }^{*} \mathrm{M}$ Gottfredsson, ${ }^{1} \mathrm{~N}$ Wilson, ${ }^{1} \mathrm{M}$ G Baker. ${ }^{1}$ University of Otago, Wellington, New Zealand; ${ }^{2}$ University of Iceland and Landspitali University Hospital, Reykjavik, Iceland

Introduction Nations varied in their response to the 1918-1919 influenza pandemic; however, certain epidemiological characteristics of this pandemic were repeated in many locations. We aimed to compare the epidemiology and public health response to this pandemic in two island nations, on opposite sides of the globe: Iceland and New Zealand.

Methods Historical accounts in both nations were reviewed, along with more recent analysis of the pandemics impact and course.

Results Both nations experienced three pandemic waves from late 1918 onwards. The second wave exacted the largest toll in terms of mortality and peaked in 3 weeks at roughly the same time in midNovember 1918. Iceland and New Zealand had similar pandemic mortality rates (5.4 vs 5.5 per 1000) among individuals of European ethnicity. Disproportionately high pandemic mortality rates among young adults compared to pre and post pandemic years was experienced by both nations. While influenza was a notifiable disease in Iceland before the pandemic, unlike New Zealand (who delayed until mid-pandemic), officials in both nations delayed in enacting response and quarantine measures. However, there is evidence that early public health control measures in specific areas of both nations resulted in lower mortality rates.

Conclusions Our study demonstrates the consistent epidemiological characteristics of the 1918-1919 influenza pandemic; in particular the similar patterns of pandemic waves and mortality, by comparing two geographically diverse island nations. These findings highlight the importance of an early public health response and the impact it can have on the outcome of a pandemic, regardless of its virulence.

\section{P1-525 RELATIONSHIP BETWEEN SERUM ANTIOXIDANT VITAMINS AND N-TERMINAL PRO-B-TYPE NATRIURETIC PEPTIDE IN A GENERAL JAPANESE POPULATION}

\section{doi:10.1136/jech.2011.142976h.13}

${ }^{1} \mathrm{~K}$ Suzuki, ${ }^{2} \mathrm{~J}$ Ishii, ${ }^{3} \mathrm{~F}$ Kitagawa, ${ }^{3} \mathrm{~A}$ Kuno, ${ }^{4} \mathrm{Y}$ Kusuhara, ${ }^{5} \mathrm{~J}$ Ochiai, ${ }^{6} \mathrm{Y}$ Ito, ${ }^{6} \mathrm{~N}$ Hamajima, ${ }^{1} \mathrm{~T}$ Inoue. ${ }^{1}$ Department of Public Health, Fujita Health University School of Health Sciences, Toyoake, Japan; ${ }^{2}$ Department of Joint Research Laboratory of Clinical Medicine, Fujita Health University, School of Medicine, Toyoake, Japan; ${ }^{3}$ Department of Joint Research Laboratory of Clinical Medicine, Fujita Health University Hospital, Toyoake, Japan; ${ }^{4}$ Department of Medical Zoology, Fujita Health University, School of Health Sciences, Toyoake, Japan; ${ }^{5}$ Department of Medical Electronics, Fujita Health University, School of Health Sciences, Toyoake, Japan; ${ }^{6}$ Department of Preventive Medicine, Nagoya University, Graduate School of Medicine, Nagoya, Japan

Introduction Several epidemiological studies have demonstrated that carotenoid relate inversely to cardiovascular disease incidence. However few studies have been conducted on the relationship between circulating antioxidant vitamins and $\mathrm{N}$-terminal pro-brain natriuretic peptide (NT-proBNP), a biomarker of cardiac function and heart failure, in the general population. The aim of this study was to investigate whether circulating antioxidant vitamins were independently related to NT-pro-BNP in a general Japanese population. Methods Subjects were 1056 inhabitants (390 men and 666 women, mean age: $60.3 \pm 10.5$ years) of Japan, who attended health check-up examinations from 2003 to 2004. Serum levels of carotenoids, retinol and tocopherols were separately determined by high-performance liquid chromatography. Serum NT-proBNP levels were measured by electrochemiluminescence immunoassay.
Results Geometric mean of serum NT-proBNP was significantly higher in women than in men (53.1 vs $39.1 \mathrm{pg} / \mathrm{ml}, \mathrm{p}<0.001)$. Partial correlation analysis, adjusting for age, smoking habits, drinking habits, serum triglyceride levels, haemoglobin A1c, body mass index, systolic blood pressure, and estimated glomerular filtration rate, revealed a significant association between serum NT-proBNP levels and serum levels of retinol $(r=-0.157, p<0.001)$, canthaxanthin $(\mathrm{r}=-0.142, \mathrm{p}<0.001)$, lycopene $(\mathrm{r}=-0.106, \mathrm{p}=0.007), \alpha$-carotene $(\mathrm{r}=-0.103, \mathrm{p}=0.009)$, and $\beta$-carotene $(\mathrm{r}=-0.086, \mathrm{p}=0.028)$ in women. In men, serum levels of antioxidant vitamins were not significantly related with serum NT-proBNP levels.

Conclusion Serum levels of retinol and several carotenoids were inversely associated with serum NT-proBNP levels even after adjustment for possible confounding factors in Japanese women, whereas no significant association was observed in Japanese men.

\section{P1-526 INCIDENCE OF VENOUS THROMBOEMBOLISM IN RELATION TO BODY MASS INDEX, POSTOPERATIVELY AND WITHOUT SURGERY}

doi:10.1136/jech.2011.142976h.14

${ }^{1,2}$ S Sweetland, ${ }^{*} 1,3$ L Parkin, ${ }^{1,2} \mathrm{~A}$ Balkwill, ${ }^{1,2} \mathrm{~V}$ Beral. ${ }^{1}$ Cancer Epidemiology Unit, University of Oxford, Oxford, UK; ${ }^{2}$ Dunedin School of Medicine, University of Otago, Dunedin, New Zealand; ${ }^{3}$ On behalf of the Million Women Study Collaborators, UK

Introduction Surgery is a major risk factor for venous thromboembolism. The incidence of venous thromboembolism is also known to increase with increasing body mass index (BMI). We describe the risk of hospital admission for (or death from) venous thromboembolism in relation to BMI, both in the absence of surgery and in the 12 weeks after surgery.

Methods Women in the UK were recruited into the Million Women Study in 1996-2001 and followed by record linkage to routinely collected inpatient and day case NHS hospital admissions and deaths. The incidence of hospital admission or death for venous thromboembolism was estimated in relation to BMI both with and without surgery.

Results 1.2 million women with an average BMI of $26.2 \mathrm{~kg} / \mathrm{m}^{2}$ were included in these analyses, $55 \%$ of whom had at least one hospital admission for surgery during follow-up. The RR of venous thromboembolism increased with increasing BMI both with and without surgery. In the absence of surgery those of BMI $\geq 35 \mathrm{~kg} / \mathrm{m}^{2}$ were 3.5 times more likely to be diagnosed with venous thromboembolism than those of BMI 22.5-24.9 kg/m² [RR 3.5 (95\%CI 3.1 to 3.9)]. A similar relationship was observed in the 12 weeks following day or inpatient surgery, but the absolute risks associated with BMI were much greater following surgery.

Conclusions The risk of venous thromboembolism increases with increasing BMI and the associated excess risk is much greater in the 12 weeks following surgery than without surgery.

\section{P1-527 STABILITY AND VARIATION IN ALCOHOL HABITS FOR DEMOGRAPHIC SUBGROUPS IN STOCKHOLM COUNTY, SWEDEN: A LONGITUDINAL STUDY 1998-2010}

doi:10.1136/jech.2011.142976h.15

L Sydén, * P Wennberg, Y Forsell, A Romelsjö. Karolinska Institutet, Stockholm, Sweden

Introduction The typical drinking pattern over the life course begins with a debut in the teenage-years, followed by increased consumption until early adulthood, and thereafter gradually decreased consumption. However, more recent cohorts show a slower decline in alcohol habits with increasing age. Given the progressing ageing of the population there is a need to understand 